the

it:

\title{
abdus salam
}

international

centre

for theoretical

physics

INFLUENCE OF NONLINEAR COUPLING

OF PULSES ON SPATIO-TEMPORAL

COMPRESSION

Monika E. Pietrzyk 
The method of spatio-temporal pulse compression takes an advantage of simultaneous spatio-temporal self-focusing of pulses propagating in anomalous or normal dispersion regime in Kerr-type bulk media or planar waveguides $[1,2,3]$. In the case of anomalous dispersion large compression factor, that is short pulses with high intensity of the optical field, can be obtained [4]. However, too high optical fields can damage the propagation medium. Therefore the process of self-focusing is ought to be suppressed at the corresponding high intensities. This can be achieved by the saturation-type nonlinearity which replaces the simplest Kerr nonlinearity [5]. Besides, it was shown recently [6] that catastrophic self-focusing of a pulse propagating in anomalous dispersion regime in a Kerr-type planar waveguide can be prevented by a presence of a second pulse which propagates in normal dispersion regirne. In [6] catastrophic self-focusing and spatio-temporal splitting in the above configuration were considered. In this paper we will focus on compression of a pulse propagating in anomalous dispersion regime in the same configuration.

To describe the propagation of the pulses we use two coupled nonlinear Schrödinger equations:

$$
\begin{gathered}
i \frac{\partial}{\partial \zeta} \Psi_{1}+\frac{1}{2} \sigma_{1} \frac{\partial^{2}}{\partial \tau^{2}} \Psi_{1}+\frac{1}{2} \frac{\partial^{2}}{\partial \xi^{2}} \Psi_{1}+\left(\left|\Psi_{1}\right|^{2}+2\left|\Psi_{2}\right|^{2}\right) \Psi_{1}=0, \\
i \frac{\partial}{\partial \zeta} \Psi_{2}+\frac{1}{2} \sigma_{2} \frac{\partial^{2}}{\partial \tau^{2}} \Psi_{2}+\mu \frac{1}{2} \frac{\partial^{2}}{\partial \xi^{2}} \Psi_{2}+r\left(\left|\Psi_{2}\right|^{2}+2\left|\Psi_{1}\right|^{2}\right) \Psi_{2}=0,
\end{gathered}
$$

where $\zeta$ is the longitudinal coordinate, $\xi=x / w_{1}$ is the spatial transverse coordinate, $\tau=\left(t-\beta_{1}^{(1)} z\right) / t_{1}$ is the local time, the parameters $\sigma_{j}, \mu, r$ denote respectively the dispersion-to-diffraction ratio, the ratio of the Fresnel diffraction length of the anomalous pulse to the Fresnel diffraction length of the normal pulse, and the ratio of the carrier frequency of the anomalous pulse to the carrier frequency of the normal pulse. In this notation $j=1,2$, where the subscript $j=1(j=2)$ refers to the pulse propagating in anomalous (normal) dispersion regime, which means that $\sigma_{1}>0$ and $\sigma_{2}<0$.

In this paper, the difference of the group velocities of the pulses and the four wave mixing terms are neglected, i.e. no energy exchange between pulses is considered. The following analysis is based on the variational method [7] and numerical simulations using the split-step spectral method [8]. Throughout the paper the pulse propagating in anomalous (normal) dispersion regime will be referred to as the anomalous (normal) pulse.

As the initial condition and the trial function in the variational method we take the Gaussian function

$$
\Psi_{j}(\zeta, \tau, \xi)=\sqrt{\kappa_{j}(\zeta)} \exp \left[-\frac{\tau^{2}\left(1+i C_{\tau j}(\zeta)\right)}{2 w_{\tau j}(\zeta)}\right] \exp \left[-\frac{\xi^{2}\left(1+i C_{\xi j}(\zeta)\right)}{2 w_{\xi j}(\zeta)}\right]
$$


where $\sqrt{\kappa_{j}(\zeta)}$ is an amplitude, $w_{\tau j}(\zeta)\left(w_{\xi j}(\zeta)\right)$ is the temporal (spatial) width and $C_{\tau j}(\zeta)\left(C_{\xi j}(\zeta)\right)$ is the temporal (spatial) chirp of the $\mathrm{j}$-th pulse, $j=1,2$. Initial conditions are defined in such a way that $w_{\tau 1}(0)=w_{\tau 2}(0)=w_{\xi 1}(0)=$ $w_{\xi 2}(0)=1, C_{\tau 1}(0)=C_{\tau 2}(0)=C_{\xi 1}(0)=C_{\xi 2}(0)=0$. Parameters $\kappa_{1}(0)$, $\kappa_{2}(0)$, are the strengths of nonlinearity.

The variational method yields the following equations describing the evolution of temporal and spatial widths of the pulses [6]:

$$
\begin{aligned}
\frac{d^{2} w_{\tau 1}}{d \zeta^{2}} & =\frac{\sigma_{1}^{2}}{w_{\tau 1}^{3}}-\sigma_{1} \frac{1}{2} \frac{\mathcal{I}_{1}}{w_{\tau 1}^{2} w_{\xi 1}}-\frac{4 \mathcal{I}_{2} w_{\tau 1} \sigma_{1}}{\left(w_{\tau 1}^{2}+w_{\tau 2}^{2}\right)^{\frac{3}{2}}\left(w_{\xi 1}^{2}+w_{\xi 2}^{2}\right)^{\frac{1}{2}}}, \\
\frac{d^{2} w_{\xi 1}}{d \zeta^{2}} & =\frac{1}{w_{\xi 1}^{3}}-\frac{1}{2} \frac{\mathcal{I}_{1}}{w_{\tau 1} w_{\xi 1}^{2}}-\frac{4 \mathcal{I}_{2} w_{\xi 1}}{\left(w_{\tau 1}^{2}+w_{\tau 2}^{2}\right)^{\frac{1}{2}}\left(w_{\xi 1}^{2}+w_{\xi 2}^{2}\right)^{\frac{3}{2}}}, \\
\frac{d^{2} w_{\tau 2}}{d \zeta^{2}} & =\frac{\sigma_{2}^{2}}{w_{\tau 2}^{3}}-\sigma_{2} \frac{1}{2} \frac{\mathcal{I}_{2} r}{w_{\tau 2}^{2} w_{\xi 2}}-\frac{4 \mathcal{I}_{1} w_{\tau 2} \sigma_{2} r}{\left(w_{\tau 1}^{2}+w_{\tau 2}^{2}\right)^{\frac{3}{2}}\left(w_{\xi 1}^{2}+w_{\xi 2}^{2}\right)^{\frac{1}{2}}}, \\
\frac{d^{2} w_{\xi 2}}{d \zeta^{2}} & =\frac{\mu^{2}}{w_{\xi 2}^{3}}-\mu \frac{1}{2} \frac{\mathcal{I}_{2} r}{w_{\tau 2} w_{\xi 2}^{2}}-\frac{4 \mathcal{I}_{1} w_{\xi 2} \mu r}{\left(w_{\tau 1}^{2}+w_{\tau 2}^{2}\right)^{\frac{1}{2}}\left(w_{\xi 1}^{2}+w_{\xi 2}^{2}\right)^{\frac{3}{2}}}
\end{aligned}
$$

The variational analysis and numerical simulations of equations (1a) and (1b) were already presented in [6] where it was shown that catastrophic selffocusing of the pulse propagating in anomalous dispersion regime can be prevented by the pulsepropagating in normal dispersion regime if the strength of nonlinearity of the latter pulse is sufficiently large, i.e.

$$
\kappa_{2}>\kappa_{t h 2}
$$

and the dispersion-to-diffraction ratio satisfies the relation

$$
\left|\sigma_{l 2}\left(\kappa_{2}\right)\right|<\left|\sigma_{2}\right|<\left|\sigma_{u 2}\left(\kappa_{2}\right)\right| \text {. }
$$

Note that values of the parameters $\kappa_{t h 2}, \sigma_{t 2}$ and $\sigma_{u 2}$ given by the variational method are different from those obtained in numerical simulations. In this paper we will concentrate on the case when the above mentioned conditions are satisfied (equations (4a)and (4b)) and study possibilities of compression of the pulse propagating in anomalous dispersion regime.

Let us first consider the evolution of the temporal and the spatial widths of the pulses. For this purpose, let us compare (see figure 1(a) and (b)) the numerical solution of differential equations (3a) - (3d) and the results of direct numerical simulations of NSEs (equation (1)). From figure 1(a) it is evident that the widths of the anomalous pulse initially decrease reaching minimal values at a certain value of the longitudinal coordinate, $\zeta_{v 2}\left(\zeta_{n 2}\right)$, remain constant in the interval

$$
\left[\zeta_{v 2} ; \zeta_{v 2}+\Delta \zeta_{v 2}\right]\left(\left[\zeta_{n 2} ; \zeta_{n 2}+\Delta \zeta_{n 2}\right]\right)
$$


and then increase. Here the subscripts $v 2$ and $(n 2)$ denote, respectively, values obtained from the variational method (the numerical simulations) for the case of two pulses, anomalous and normal, propagating simultaneously. Note that $\Delta \zeta_{n 2}>\Delta \zeta_{v 2}$ and $\Delta \zeta_{n 2}>\Delta \zeta_{n 1}$, where $\Delta \zeta_{n 1}$ is the corresponding interval obtained from the numerical simulations for a single pulse with anomalous dispersion propagating in a Kerr-type planar waveguide (see [9]).

In figure 1(b) the evolution of the temporal and spatial width of the normal pulse is displayed. It is seen that the discrepancy between the results of the numerical and the variational method is more significant than in the case of the anomalous pulse. Namely, the numerical simulations indicate that an initial narrowing of the spatial and the temporal width of the pulse and spatio-temporal splitting occur, while the variational estimation overlooks these effects. Note that a similar discrepancy also occurs in the case of a single pulse propagating in normal dispersion regime in a nonlinear planar waveguide [9].

The next step in our study is to consider details of the compression of the anomalous pulse. Compression is characterized by the maximal compression factor, $c_{\max }$, which is defined as the ratio of the initial temporal width of the pulse to the minimal temporal width which can be achieved during the propagation of the pulse [1]. Since the minimal temporal width of the anomalous pulse is reached when the condition given by equation (5) is satisfied, then for the optimization of a compressor, i.e. to achieve the largest possible compression, the length of the planar waveguide in which the pulses propagate should also satisfy this condition. Therefore, the longer is the interval $\Delta \zeta_{n 2}$, the easier is to calibrate the compressor. From this point of view the compressor with two simultaneously propagating pulses seems to be more beneficial than the compressor with a single pulse, since $\Delta \zeta_{n 2}>\Delta \zeta_{n 1}$ (see the discussion above).

In figure 2 the dependence of the maximal compression factor of the anomalous pulse, $c_{\max }$, on the dispersion-to-diffraction ratio of the nomal pulse, $\sigma_{2}$, obtained with the aid of the variational method is displayed. Three different values of the strength of nonlinearity of the anomalous pulse are considered: $\kappa_{1}=1.9<\kappa_{\text {cat } v}, \kappa_{1}=2.0=\kappa_{\text {cat } v}$, and $\kappa_{1}=2.1>\kappa_{\text {cat } v}$, where $\kappa_{\text {cat } v}$ is the threshold of catastrophic self-focusing given by the variational method for a single pulse propagating in anomalous dispersion regime [10]. It is seen that for the first two cases represented, respectively, by full and broken curve in figure 2, the parameter $c_{\max }$ increases with the decreasing of $\sigma_{2}$, and reaches a maximal value for $\sigma_{2} \rightarrow 0$. Another situation takes place for the strength of nonlinearity exceeding the critical value, $\kappa_{1}>\kappa_{\text {cat }}=2.0$, (see chain curve in figure 2). Namely, we see two maxima in the dependence of the parameter $c_{\max }$ on $\sigma_{2}$ : one at $\sigma_{2} \rightarrow \sigma_{l 2}$ and the second one at $\sigma_{2} \rightarrow \sigma_{u 2}$. Here $\sigma_{l 2}, \sigma_{u 2}$ are the thresholds of catastrophic self-focusing (see equation (j)). 
a)

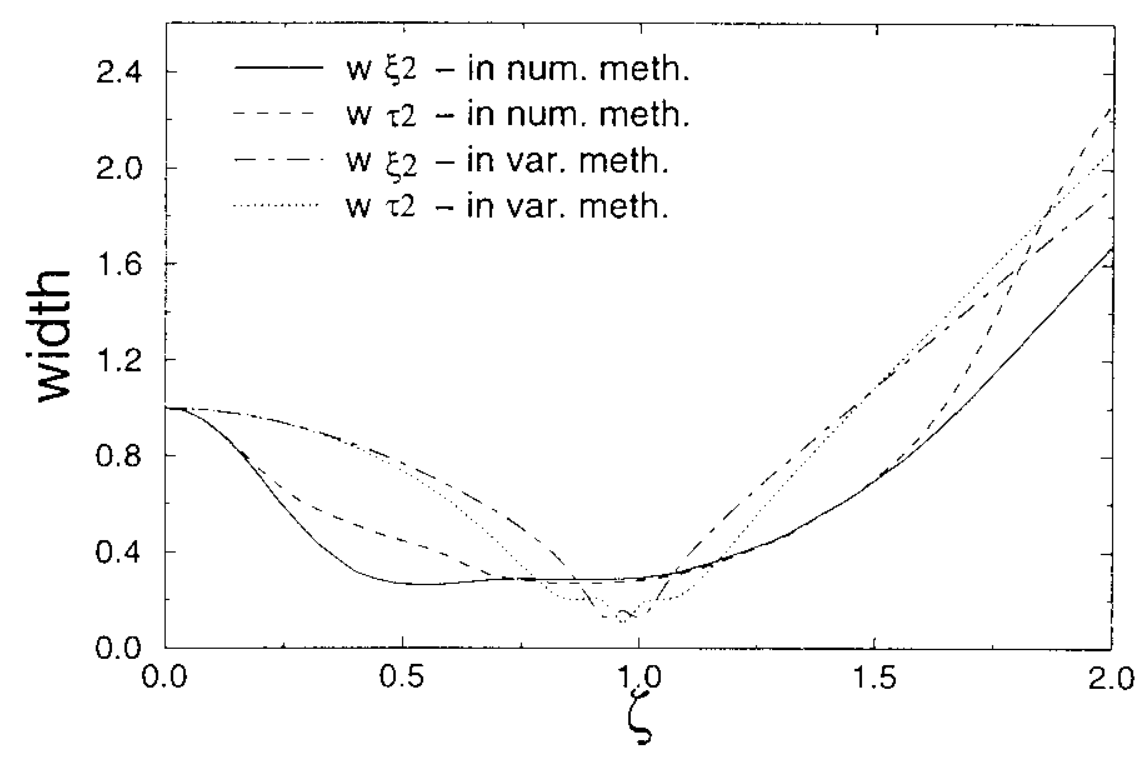

b)

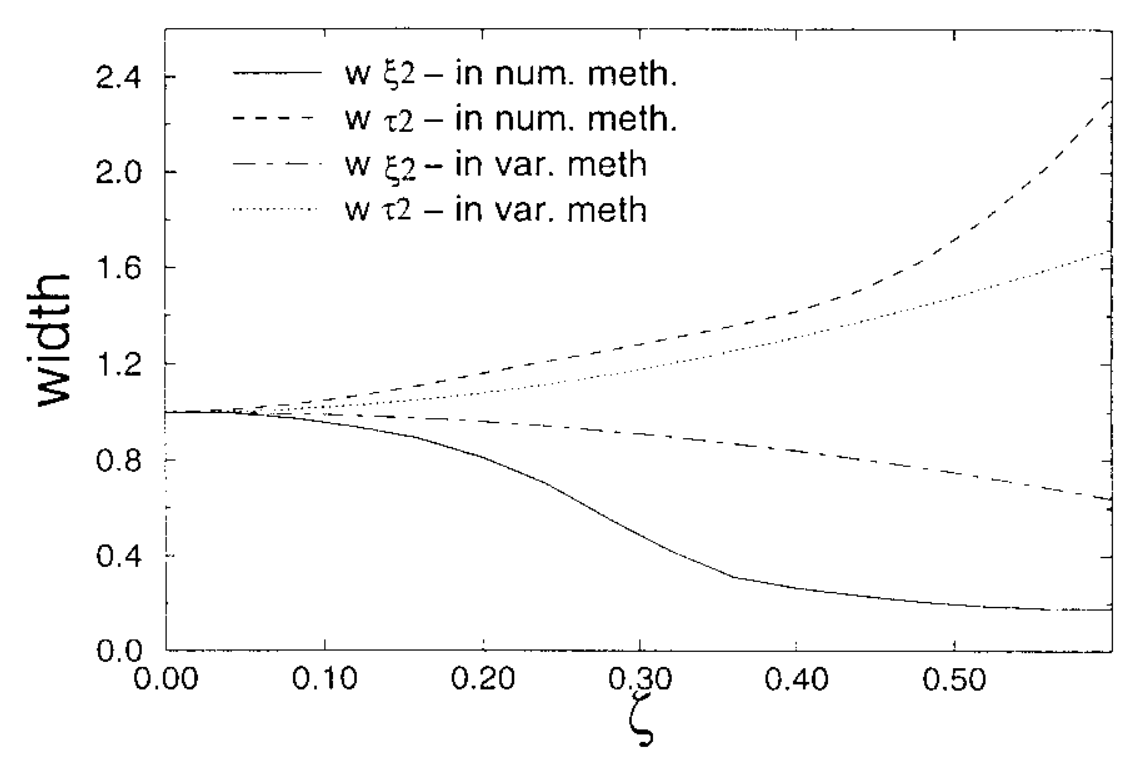

Figure 1: Comparison of the results obtained from the variational method and the numerical simulations regarding the evolution of the temporal and the spatial width of the anomalous (a) and the normal pulse (b) in the case of their simultaneous propagation $\sigma_{1}=1, \kappa_{1}=2, \sigma_{2}=-1, \kappa_{2}=2$. 


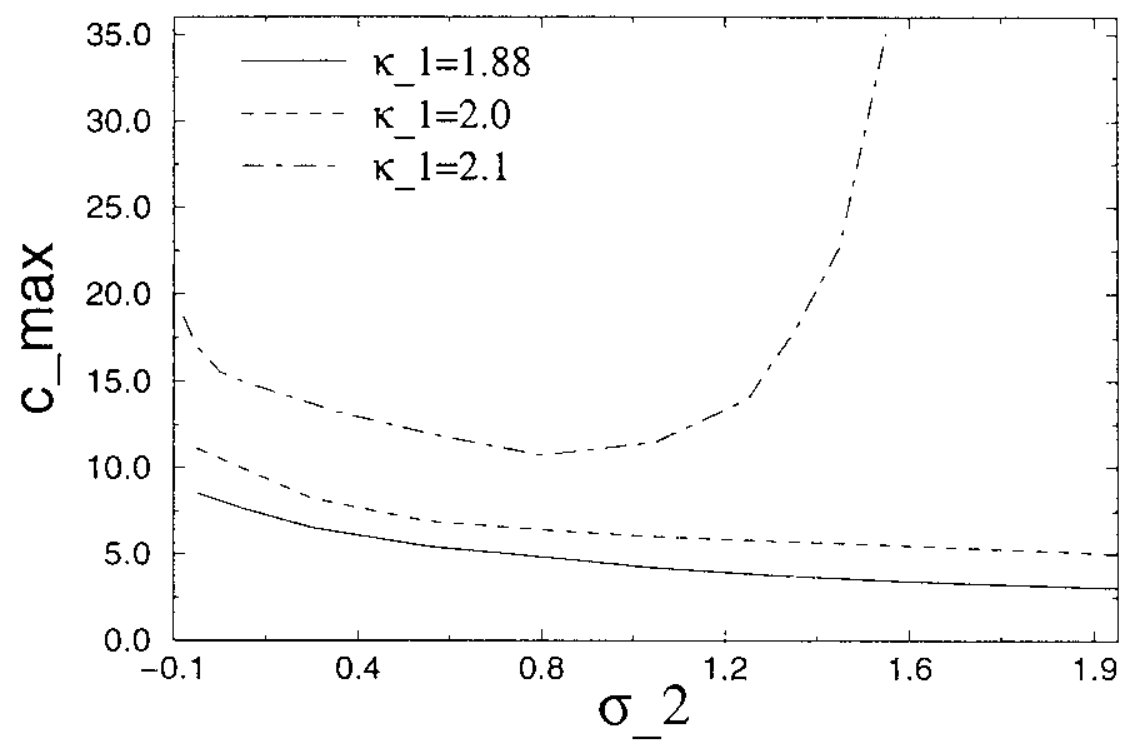

Figure 2: The maximal compression factor of the anomalous pulse, $c_{\max }$, versus the dispersion-to-diffraction ratio of the normal pulse, $\sigma_{2}$, obtained with the aid of the variational method. Three different values of the strength of nonlinearity of the anomalous pulse are considered: $\kappa_{1}=1.88<\kappa_{\text {crit } v}$ (full curve), $\kappa_{1}=2.0=\kappa_{\text {crit } v}$ (broken curve), and $\kappa_{1}=2.1=\kappa_{\text {crit } v}$ (chain curve), where $\kappa_{\text {crit }} v$ is the threshold of catastrophic self-focusing given by the variational method for the case of a single pulse propagating in anomalous dispersion regime, $\sigma_{1}=\sigma_{2}=1, \kappa_{2}=1.0$.

From figure 2 it also follows that the maximum compression factor increases with the strength of nonlinearity, i.e. the relation

$$
c_{\max }\left(\kappa_{1}>\kappa_{\text {cat }}\right)>c_{\text {max }}\left(\kappa_{1}<\kappa_{\text {cat } v}\right)
$$

is satisfied.

The results of numerical simulations displayed in figure 3 manifest qualitative similarity to the described above variational estimations. Namely, for small values of the strength of nonlinearity of the anomalous pulse, $\kappa_{1}<\kappa_{\text {cat } n}$, (see full circle points in figure 3) there is only one maximum in the dependence of the parameter $c_{\max }$ on $\sigma_{2}$, whereas for higher values of nonlinearity, $\kappa_{1}>\kappa_{\text {cat } n}$, (see empty square points in figure 3 ) two maxima arise: for $\sigma_{2} \rightarrow \sigma_{l 2}$ and for $\sigma_{2} \rightarrow \sigma_{u 2}$. Moreover, the relation (6) obtained by the variational method is also satisfied, one should only replace $\kappa_{\text {cat } n}$ by the corresponding quantity obtained by numerical simulations, $\kappa_{\text {cat } n}=1.89$ [9]. Therefore, in order to increase compression one should choose the parameters of the system in such a way that $\kappa_{1}>\kappa_{\text {cat }}$ and that either $\sigma_{2} \rightarrow \sigma_{12}$ or $\sigma_{2} \rightarrow \sigma_{u 2}$.

Concluding, in this paper the details concerning spatio-temporal compression of a pulse in anomalous dispersion regime, which propagates in a nonlinear planar waveguide simultaneously with a pulse in normal dispersion regime were studied. The influence of the dispersion-to-diffraction ratio 


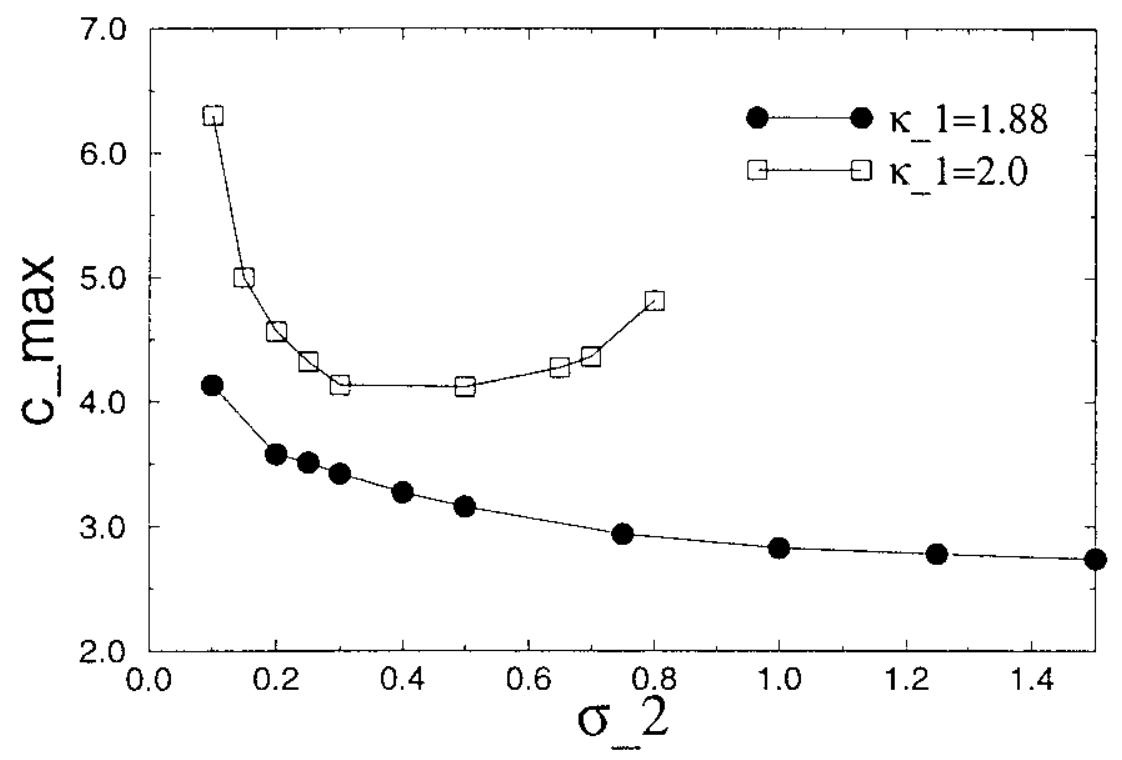

Figure 3: The maximal compression factor of the anomalous pulse, $c_{\max }$, versus the dispersion-to-diffraction ratio of the normal pulse, $\sigma_{2}$, obtained from the numerical simulations. Two different values of the strength of nonlinearity of the anomalous pulse are considered: $\kappa_{1}=1.88<\kappa_{\text {crit }}$ (full circles), and $\kappa_{1}=2.0>\kappa_{\text {crit } v}$ (empty boxes), where $\kappa_{c r i t v}=1.89$ is the threshold of catastrophic self-focusing given by the numerical simulations for the case of a single pulse propagating in anomalous dispersion regime, $\sigma_{1}=\sigma_{2}=1, \kappa_{2}=1.0$.

of the pulse propagating in normal dispersion regime and of the strength of nonlinearity of the pulse propagating in anomalous dispersion regime on the maximal compression factor of the latter pulse were considered. It was shown by the variational estimations and confirmed by numerical simulations that in order to optimize a compressor, i.c. to achieve the largest possible compression one should have a sufficiently large strength of nonlinearity of the pulse propagating in anomalous dispersion regime and either sufficiontly small or sufficiently large dispersion-to-diffraction ratio of the pulse propagating in normal dispersion regime. The length of the waveguide in which pulses propagate should be, as well, suitably chosen. It was also found that the configuration of the compressor with two simultaneously propagating pulses seems to be more beneficial than the configuration with a single pulse.

\section{Acknowledgments}

The work was supported by the Polish Committee of Scientific Research (KBN, grant no 8T11F 007 14) and the Deutsche Akademische Austauschdienst (DAAD) to both of which I express my gratitude. Guest Scientist (the DAAD fellowship, 01.19.1998 - 31-07-1999). The numerical calculations were partially performed at the Abdus Salam International Centre for Theoretical Physics, Trieste, Italy. I gratefully acknowledge the Director of the Centre, Prof. M. Virasoro and Prof. G. Denardo for their kind hospitality and helpful support. 


\section{References}

[1] A. T. Ryan and G. P. Agrawal, Opt. Lett. 20, 306 (1995).

[2] A. T. Ryan and G. P. Agrawal, J. Opt. Soc. Am. B. 12, 2382 (1995).

[3] M. Pietrzyk, Opt. and Q.E. 29, 579 (1997).

[4] Y. Silberberg, Opt. Lett. 15, 1282 (1990).

[5] M. Karlsson, Phys. Rev. A 46, 2726 (1992).

[6] M. Pietrzyk, "On the properties of two pulses propagating simultaneously in different dispersion regimes in a nonlinear planar waveguide," submitted for publication .

[7] D. Anderson and M. Bonnedal, Phys. Fluids 22, 105 (1979).

[8] G. P. Agrawal, "Nonlinear Fiber Optics" (Academic Press, London, 1989).

[9] M. Pietrzyk, "Optical pulse propagation and compression in a planar dispersive Kerr-type nonlinear medium," PhD thesis, (in polish) .

[10] M. Desaix, D. Anderson, and M. Lisak, J.Opt.Soc.Am. B. 8, 2082 (1991). 